\title{
LOW VOLUME FEMORAL NERVE BLOCK AND "INTERSPACE BETWEEN THE POPLITEAL ARTERY AND POSTERIOR CAPSULE OF THE KNEE" (IPACK) BLOCK FOR TOTAL KNEE ARTHROPLASTY
}

M.P. Sebastian ${ }^{1}$, J. Parry ${ }^{1}$, S. Nadaraja ${ }^{1}$, A. Sell ${ }^{1}$, R. Sharma ${ }^{1}$, V. Ramesh ${ }^{1}$.

1. Royal National Orthopaedic Hospital, Anaesthetics, Stanmore, United Kingdom.

\section{Background and Aims}

Total knee arthroplasty (TKA) is one of the most commonly performed and painful orthopaedic procedures. Femoral nerve block (FNB) is proven to provide effective analgesia, however the use of high doses or continuous infusion are often associated with delayed ambulation ${ }^{1}$. IPACK block is a novel technique shown to improve pain relief whilst preserving motor function ${ }^{1}$. We examined the impact on mobilisation and postoperative pain of a multimodal analgesia protocol using a single low dose FNB and IPACK block.

\section{Methods}

After local institutional approval, a retrospective patient chart review was conducted. Inclusion criteria included adult patients undergoing TKA and having an ultrasound guided FNB using $10 \mathrm{ml} 0.25 \%$ levobupicaine and IPACK block using $20 \mathrm{ml} 0.25 \%$ levobupicaine as part of an established multimodal analgesia protocol. The primary outcome was ambulation within the first 24 hours. Secondary outcomes included numerical pain scale scores (NPSS), time for first morphine request, opioid consumption and time to physiotherapy discharge.

\section{Results}

Eight patients were included. All were able to mobilise within 24 hours. Median pain scores were $0(0-3)$ day $0,2.25(0-5)$ day 1 and $2(0-4)$ day 2 . Two patients did not need any morphine during admission. Among the others, median time for first request of morphine was 17 hours (8-41). Median oral morphine consumption within 48 hours was $10 \mathrm{mg}(0-110)$. Median duration for physiotherapy discharge was 2.5 days (1-7).
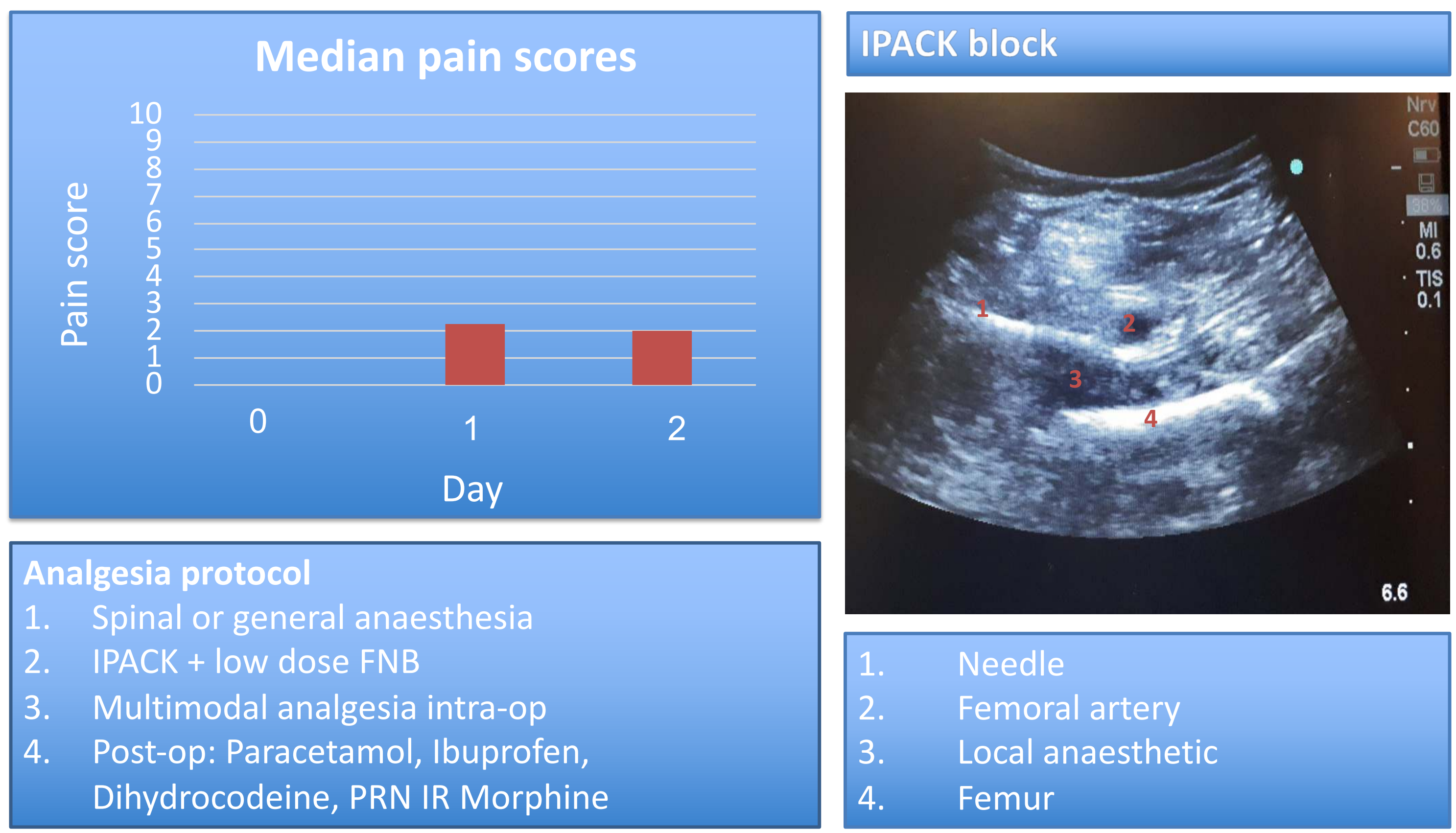

\section{Conclusion}

The combination of a low dose FNB and IPACK block as part of a multimodal analgesia protocol allowed early ambulation and provided low pain scores with low opioid consumption in our patients. 